\title{
Instrumentación rotatoria comparado con instrumentación manual para tratamiento endodóntico en dientes permanentes.
}

\section{Rotary instrumentation compared to manual instrumentation for endodontic treatment in permanent teeth.}

\begin{abstract}
Sebastian Wall ${ }^{1,2}$, Sofía Maureira ${ }^{1,2}$, Carla Madrid ${ }^{1,2}$, Carola Antini $^{1,2^{*}}$
1. Escuela de Odontología, Facultad de Medicina, Pontificia Universidad Católica de Chile, Santiago, Chile.

2. Proyecto Epistemonikos, Santiago, Chile.

* Correspondencia Autor: Carola Antini | Dirección: Centro Evidencia UC, Pontificia Universidad

Católica de Chile, Diagonal Paraguay 476,

Santiago, Chile | E-mail: cantini@uc.cl

RESUMEN

Introducción:El éxito del tratamiento endodóntico requiere de la desinfección completa del sistema de canales radiculares. Convencionalmente este procedimiento se realiza a través de maniobras de instrumentación rotatoria o manual e irrigación química. Dentro de las diferentes técnicas de instrumentación, la de tipo rotatoria ha surgido como una alternativa a la instrumentación manual, cuyos beneficios en comparación a ésta aún deben ser dilucidados. Métodos: Realizamos una búsqueda en Epistemonikos, la mayor base de datos de revisiones sistemáticas en salud, la cual es mantenida mediante el cribado de múltiples fuentes de información, incluyendo MEDLINE, EMBASE, Cochrane, entre otras. Extrajimos los datos desde las revisiones identificadas, analizamos los datos de los estudios primarios, realizamos un metanálisis y preparamos una tabla de resumen de los resultados utilizando el método GRADE. Resultados y conclusiones: Identificamos cinco revisiones sistemáticas que en conjunto incluyeron 15 estudios primarios, de los cuales, 10 corresponden a ensayos aleatorizados. Concluimos que el uso de instrumentación rotatoria en comparación a instrumentación manual probablemente disminuye la incidencia del dolor postoperatorio. Además, la instrumentación rotatoria podría disminuir el uso de analgésicos post tratamiento endodóntico. Sin embargo, podría resultar en poca o nula diferencia en la intensidad del dolor, pero la certeza de la evidencia es baja. Además, no es posible establecer con claridad si el uso de instrumentación rotatoria en comparación a la instrumentación manual aumenta la reparación apical debido a que la certeza de la evidencia existente ha sido evaluada como muy baja.
\end{abstract}

\section{PALABRAS CLAVE}

Tratamiento endodóntico; Instrumentación rotatoria; instrumentación manual; Epistemonikos; GRADE.

\section{ABSTRACT}

Introduction: Successful root canal therapy (endodontic treatment) requires complete disinfection of the root canal system. Traditionally, disinfection of the root canal system involves rotary or manual instrumentation and chemical irrigation. Various rotary instrumentation techniques have emerged as an alternative to manual instrumentation, but its benefits against manual techniques need to be clarified. Methods: We searched in Epistemonikos, the largest database of systematic reviews in health, which is maintained by screening multiple information sources, including MEDLINE, EMBASE, Cochrane, among others. We extracted data from the systematic reviews, reanalyzed data of primary studies, conducted a meta-analysis and generated a summary of findings table using the GRADE approach. Results and conclusions: We identified five systematic reviews that together included 15 primary studies, of which 10 correspond to randomized trials. We conclude that the use of rotary instrumentation compared to manual instrumentation probably reduces the incidence of pain. Also, rotatory instrumentation may reduce the use of postoperative analgesics. However, it could result in little or no difference in pain intensity, but the certainty of the evidence is low. Furthermore, it is not possible to clearly establish whether the use of rotary instrumentation increases apical repair as the certainty of the evidence has been assessed as very low.

\section{KEY WORDS}

Endodontic treatment; Rotary instrumentation; manual instrumentation; Epistemonikos; GRADE.

Int. J. Inter. Dent Vol. 14(1); 67-72, 2021. 


\section{PROBLEMA}

Las patologías pulpo periodontales suelen presentarse como consecuencia del curso natural de las lesiones cariosas y traumatismos dentoalveolares. Estos cuadros pueden tener un curso de evolución rápido o lento, manifestándose como patologías agudas o crónicas $^{(1)}$. Considerando la importancia de realizar en el diente un tratamiento rehabilitador con pronóstico favorable, es primordial el tratamiento oportuno y eficaz, asociado a una terapia endodóntica apropiada(2).

Dentro de las etapas del tratamiento endodóntico, es fundamental lograr una adecuada preparación químico mecánica, donde se busca la desinfección y conformación del sistema de canales radiculares ${ }^{(3)}$. Mediante las nuevas tecnologías en endodoncia, se ha incorporado el uso de técnicas de instrumentación rotatoria en la fase de preparación químico mecánica, las que presentan ventajas y desventajas en comparación con las técnicas de instrumentación manual, además de una curva de aprendizaje mayor(4). Este resumen busca evaluar la efectividad y seguridad de la instrumentación rotatoria en comparación a la instrumentación manual para tratamientos de endodoncia en dientes permanentes.

\section{MÉTODOS}

Realizamos una búsqueda en Epistemonikos, la mayor base de datos de revisiones sistemáticas en salud, la cual es mantenida mediante búsquedas en múltiples fuentes de información, incluyendo MEDLINE, EMBASE, Cochrane, entre otras. Extrajimos los datos desde las revisiones identificadas y analizamos los datos de los estudios primarios. Con esta información, generamos un resumen estructurado denominado FRISBEE (Friendly Summaries of Body of Evidence using Epistemonikos), siguiendo un formato preestablecido, que incluye mensajes clave, un resumen del conjunto de evidencia (presentado como matriz de evidencia en Epistemonikos), metanálisis del total de los estudios cuando sea posible, una tabla de resumen de resultados con el método GRADE y una sección de otras consideraciones para la toma de decisión.

\section{Mensajes clave}

- El uso de instrumentación rotatoria en comparación a instrumentación manual probablemente disminuye la incidencia de dolor postoperatorio.

- El uso de instrumentación rotatoria en comparación a instrumentación manual podría resultar en poca o nula diferencia en la intensidad de dolor postoperatorio medido a las 6 u 8 horas y a 24 horas (certeza de la evidencia baja).

- La instrumentación rotatoria podría disminuir el uso de analgésicos post tratamiento endodóntico (certeza de la evidencia baja).

- No existe claridad de si el uso de instrumentación rotatoria en comparación a la instrumentación manual aumenta la reparación apical en tanto la certeza de la evidencia ha sido evaluada como muy baja.

- No se encontró evidencia evaluando la sobrevida de diente en boca e incidencia de errores o accidentes intraoperatorios.

\section{Acerca del conjunto de evidencia para esta pregunta}

Encontramos cinco revisiones sistemáticas ${ }^{(5,6,7,8,9)}$ que incluyeron 15 estudios primarios $(10,11,12,13,14,15,1$ $6,17,18,19,20,21,22,23,24)$, de los cuales, 10

Cuál es la evidencia

Véase matriz de evidencia en Epistemonikos más abajo. corresponden a ensayos aleatorizados $(10,11,12,13,14,15,16,17,18,19)$.

Esta tabla y el resumen en general se basa en estos últimos, dado que los estudios observacionales no aumentaban la certeza de la evidencia existente, ni entregaban información adicional relevante.

Todos los ensayos incluyeron pacientes con dentición permanente que requerían tratamiento endodóntico, sin restricción por tipo de diente o diagnóstico pulpar y

Qué tipo de pacientes incluyeron los estudios*

Qué tipo de intervenciones incluyeron los estudios* midieron periapical $^{(10,11,12,13,14,15,16,17,18,19)}$. Cinco ensayos incluyeron molares inferiores $(10,11,16,18,19)$ y cuatro ensayos incluyeron dientes con canal único ${ }^{(12,13,14,15)}$. Solo un ensayo no reportó el tipo de dientes incluidos ${ }^{(17)}$.

Todos los ensayos compararon instrumentación rotatoria de giro continuo con instrumentación manual. No fueron incluidos los ensayos que compararon instrumentación rotatoria reciprocante.

Cuatro ensayos evaluaron instrumentación rotatoria ProTaper $(10,14,15,19)$; uno evaluó instrumentación rotatoria Race(11); un ensayo evaluó instrumentación rotatoria Profile ${ }^{(12)}$; uno evaluó instrumentación rotatoria Mtwo ${ }^{(13)}$; un ensayo evaluó instrumentación rotatoria One Shape ${ }^{(16)}$; uno evaluó instrumentación rotatoria Hero642(17) y un ensayo evaluó instrumentación rotatoria con $\mathrm{NiTi}^{(19)}$.

En la instrumentación manual, ocho ensayos utilizaron limas $\mathrm{K}^{(10,12,13,14,15,16,18,19)}$, uno utilizó limas K-Flexofiles ${ }^{(11)}$ y un ensayo utilizó limas Nitiflex ${ }^{(17)}$.

Los ensayos reportaron múltiples desenlaces, los cuales fueron agrupados por las revisiones sistemáticas de la siguiente manera:

- Incidencia de dolor postoperatorio

- Intensidad de dolor postoperatorio (Escala Visual Análoga)

- Uso de analgésicos

- Reparación apical

Las revisiones identificadas no reportaron el seguimiento de los ensayos.

* La información sobre los estudios primarios es extraída desde las revisiones sistemáticas identificadas, no directamente desde los estudios, a menos que se especifique lo contrario.

\section{RESUMEN DE LOS RESULTADOS}

La información sobre los efectos de la instrumentación rotatoria en pacientes con dentición permanente que requieren tratamiento endodóntico está basada en 10 ensayos aleatorizados que incluyeron 1237 dientes.

Cuatro ensayos midieron el desenlace incidencia de dolor postoperatorio (616 dientes) ${ }^{(12,13,17,18)}$. Dos ensayos midieron el desenlace de intensidad de dolor postoperatorio a 6 u 8 horas y 24 horas (160 dientes) $)^{(13,16)}$.

Tres ensayos midieron el desenlace uso de analgésicos (252 dientes) $(10,11,13)$. Dos estudios midieron el desenlace reparación apical (1209 dientes) $)^{(20,21)}$. Sin embargo, ninguna revisión permitió la extracción de datos de manera que pudieran ser incorporados a un metanálisis, por lo que la información de dichos desenlaces se presenta como síntesis narrativa.

El resumen de los resultados es el siguiente:

- La instrumentación rotatoria probablemente disminuye la incidencia de dolor postoperatorio.

- La instrumentación rotatoria podría resultar en poca o nula diferencia en la intensidad de dolor postoperatorio medido a las 6 u 8 horas (certeza de la evidencia baja). 
- La instrumentación rotatoria podría resultar en poca o nula diferencia en la intensidad del dolor postoperatorio medido a las 24 horas (certeza de la evidencia baja).

- La instrumentación rotatoria podría disminuir el uso de analgésicos post tratamiento endodóntico (certeza de la evidencia baja).

- No es posible establecer con claridad si la instrumentación rotatoria aumenta la reparación apical, debido a que la certeza de la evidencia existente ha sido evaluada como muy baja.

- No se encontró evidencia que evaluara la sobrevida del diente en boca.

- No se encontró evidencia que evaluara la incidencia de errores o accidentes intraoperatorios.

\begin{tabular}{|c|c|c|c|c|}
\hline \multicolumn{5}{|c|}{$\begin{array}{l}\text { Instrumentación rotatoria comparada con instrumentación manual para } \\
\text { tratamiento endodóntico en dentición permanente }\end{array}$} \\
\hline $\begin{array}{l}\text { Pacientes } \\
\text { Intervención } \\
\text { Comparación }\end{array}$ & \multicolumn{4}{|c|}{$\begin{array}{l}\text { Pacientes con dentición permanente que requieren tratamiento } \\
\text { endodóntico } \\
\text { Instrumentación rotatoria } \\
\text { Instrumentación manual }\end{array}$} \\
\hline \multirow{3}{*}{ Desenlaces } & Efecto & ssoluto* & \multirow{3}{*}{$\begin{array}{l}\text { Efecto } \\
\text { relativo } \\
(\text { IC } 95 \%)\end{array}$} & \multirow{3}{*}{$\begin{array}{l}\text { Certeza de } \\
\text { la evidencia } \\
\text { (GRADE) }\end{array}$} \\
\hline & $\begin{array}{l}\text { CON } \\
\text { instrumentación } \\
\text { manual }\end{array}$ & $\begin{array}{l}\text { CON } \\
\text { instrumentación } \\
\text { rotatoria } \\
\end{array}$ & & \\
\hline & \multicolumn{2}{|c|}{ Diferencia: dientes por 1000} & & \\
\hline \multirow{2}{*}{$\begin{array}{l}\text { Incidencia de } \\
\text { dolor } \\
\text { postoperatorio }\end{array}$} & 323 por 1000 & 84 por 1000 & \multirow{2}{*}{$\begin{array}{l}\text { RR } 0,26 \\
(0,14 \text { a } \\
0,49)\end{array}$} & \multirow{2}{*}{$\begin{array}{l}\oplus \oplus \oplus \bigcirc^{1} \\
\text { Moderada }\end{array}$} \\
\hline & \multicolumn{2}{|c|}{$\begin{array}{c}\text { Diferencia: } 239 \text { menos } \\
\text { (Margen de error: } 278 \text { a } 165 \text { menos) }\end{array}$} & & \\
\hline \multirow{2}{*}{$\begin{array}{l}\text { Intensidad de } \\
\text { dolor } \\
\text { postoperatorio a } \\
6 \text { u } 8 \text { horas** }\end{array}$} & 5 puntos & 3,03 puntos & \multirow[b]{2}{*}{--} & \multirow[b]{2}{*}{$\begin{array}{l}\oplus \oplus \bigcirc^{1,2} \\
\text { Baja }\end{array}$} \\
\hline & \multicolumn{2}{|c|}{$\begin{array}{l}\text { DM: } 1,97 \text { puntos menos } \\
\text { (Margen de error: } 3,09 \text { a } 0,86 \text { menos) }\end{array}$} & & \\
\hline \multirow{2}{*}{$\begin{array}{l}\text { Intensidad de } \\
\text { dolor } \\
\text { postoperatorio a } \\
24 \text { horas** }\end{array}$} & 2,76 puntos & 0,9 puntos & \multirow[b]{2}{*}{--} & \multirow{2}{*}{$\begin{array}{l}\oplus \bigcirc_{\text {Baja }}^{1,2} \\
\text { Baja }\end{array}$} \\
\hline & \multicolumn{2}{|c|}{$\begin{array}{l}\text { DM: } 1,86 \text { puntos menos } \\
\text { (Margen de error: } 2,54 \text { a } 1,19 \text { menos) }\end{array}$} & & \\
\hline $\begin{array}{l}\text { Uso de } \\
\text { analgésicos }\end{array}$ & \multicolumn{3}{|c|}{$\begin{array}{c}\text { Dos revisiones sistemáticas [5],[6] que se basaron en tres } \\
\text { ensayos (252 dientes) [10],[11],[13], reportaron que la } \\
\text { instrumentación rotatoria disminuye el uso de analgésicos } \\
\text { luego del tratamiento endodóntico comparado con la } \\
\text { instrumentación manual. }\end{array}$} & $\underset{\mathrm{Baja}}{\oplus \bigcirc^{1,2}}$ \\
\hline $\begin{array}{l}\text { Reparación } \\
\text { apical }\end{array}$ & \multicolumn{3}{|c|}{$\begin{array}{c}\text { Una revisión sistemática [8] que se basó en dos estudios } \\
\text { (1209 dientes) [20],[21], reportó que la instrumentación } \\
\text { rotatoria aumenta el éxito de la reparación apical luego del } \\
\text { tratamiento endodóntico comparado con la } \\
\text { instrumentación manual. }\end{array}$} & $\begin{array}{l}\oplus \bigcirc \bigcirc \bigcirc^{3,4} \\
\text { Muy baja }\end{array}$ \\
\hline $\begin{array}{l}\text { Sobrevida del } \\
\text { diente en boca }\end{array}$ & \multicolumn{2}{|c|}{$\begin{array}{l}\text { El desenlace no fue medido o reportado por } \\
\text { las revisiones sistemáticas. }\end{array}$} & -- & -- \\
\hline $\begin{array}{l}\text { Incidencia de } \\
\text { errores o } \\
\text { accidentes } \\
\text { intraoperatorios }\end{array}$ & \multicolumn{2}{|c|}{$\begin{array}{l}\text { El desenlace no fue medido o reportado por } \\
\text { las revisiones sistemáticas. }\end{array}$} & -- & -- \\
\hline
\end{tabular}


Margen de error: Intervalo de confianza del 95\% (IC 95\%).

RR: Riesgo relativo.

DM: Diferencia de medias.

GRADE: Grados de evidencia del GRADE Working Group (ver más adelante).

*Los riesgos/promedio CON instrumentación manual están basados en los riesgos/promedio del grupo control en los estudios. El riesgo/promedio CON instrumentación rotatoria (y su margen de error) está calculado a partir del efecto relativo/diferencia de medias (y su margen de error).

** Desenlace medido con Escala Visual Análoga (EVA), que va entre cero a 10 puntos, donde mayor puntaje refleja mayor dolor. Si bien no se encontraron revisiones o estudios que evaluaran la diferencia mínimamente importante (MID - Minimally Important Difference) en la población de interés, se identificó un estudio en pacientes sometidos a cirugía de terceros molares que fijó esta diferencia en 2,5 puntos [25].

${ }^{1}$ Se disminuyó un nivel de certeza de evidencia por riesgo de sesgo, dado que los ensayos presentaban problemas en la generación de la secuencia aleatoria y el ocultamiento de ésta.

${ }^{2}$ Se disminuyó un nivel de certeza de evidencia por imprecisión, ya que a cada extremo del intervalo de confianza se tomarían decisiones diferentes. En el caso del desenlace uso de analgésicos, se decidió disminuir por este motivo considerando el número de ensayos y tamaño muestral evaluado, en tanto no se puede descartar que el efecto observado sea producto del azar.

${ }^{3}$ Estudios observacionales.

${ }^{4}$ Se disminuyó un nivel de certeza de evidencia por riesgo de sesgo, dado que los estudios presentaban limitaciones asociadas al sesgo de detección (ciego de los evaluadores).

Siga el enlace para acceder a la versión interactiva de esta tabla (Interactive Summary of Findings - iSoF)

\section{Acerca de la certeza de la evidencia (GRADE)*}

\section{$\oplus \oplus \oplus \oplus$}

Alta: La investigación entrega una muy buena indicación del efecto probable. La probabilidad de que el efecto sea sustancialmente distinto ${ }^{\dagger}$ es baja.

$\oplus \oplus \oplus \bigcirc$

Moderada: La investigación entrega una buena indicación del efecto probable. La probabilidad de que el efecto sea sustancialmente distinto ${ }^{\dagger}$ es moderada.

$\oplus \oplus \bigcirc$

Baja: La investigación entrega alguna indicación del efecto probable. Sin embargo, la probabilidad de que el efecto sea sustancialmente distinto ${ }^{\dagger}$ es alta.

\section{$\oplus \bigcirc \bigcirc \mathrm{O}$}

Muy baja: La investigación no entrega una estimación confiable del efecto probable. La probabilidad de que el efecto sea sustancialmente distinto ${ }^{\dagger}$ es muy alta.

*Esto es también denominado 'calidad de la evidencia' o 'confianza en los estimadores del efecto'. tSustancialmente distinto = una diferencia suficientemente grande como para afectar la decisión 


\section{OTRAS CONSIDERACIONES PARA LA TOMA DE DECISIÓN}

\section{A quién se aplica y a quién no se aplica esta evidencia}

- Los resultados de este resumen son aplicables a pacientes con dentición permanente con cierre apical completo que presenten alguna patología endodóntica y requieran tratamiento.

- Esta evidencia no es aplicable a pacientes con dentición primaria o dientes permanentes jóvenes.

- Esta evidencia no es aplicable a la instrumentación mecanizada de tipo reciprocante.

\section{Sobre los desenlaces incluidos en este resumen}

- Los desenlaces incluidos en esta tabla son aquellos considerados críticos para la toma de decisiones de acuerdo a la opinión de los autores de este resumen y la mayoría de ellos se encuentran en concordancia con las revisiones sistemáticas identificadas.

- Los desenlaces sobrevida del diente en boca e incidencia de errores o accidentes intraoperatorios fueron considerados críticos para la toma de decisión debido a que se ha planteado que la instrumentación rotatoria al compararla con la instrumentación manual disminuiría la frecuencia de errores procedimentales dado el diseño del instrumento, pero estos no fueron medidos por las revisiones sistemáticas identificadas.

\section{Balance daño/beneficio y certeza de la evidencia}

- La evidencia muestra un posible beneficio en el uso de instrumentación rotatoria en comparación a la instrumentación manual probablemente disminuye la incidencia del dolor postoperatorio y podría disminuir el uso de analgésicos luego de la terapia endodóntica, pero la certeza de la evidencia ha sido evaluada como baja.

- Por otro lado, el uso de diferentes sistemas de limas rotatorias y la ausencia de información respecto al seguimiento de los ensayos incluidos, dificulta la medición y comparación de los desenlaces a largo plazo de ambas intervenciones.

- Con lo anterior, no es posible hacer un adecuado balance entre riesgos y beneficios de la instrumentación rotatoria por sobre la instrumentación manual debido a la falta de información existente sobre desenlaces considerados críticos.

\section{Consideraciones de recursos}

- Ninguno de las revisiones incluidas realizó un análisis de costos entre la instrumentación rotatoria y la instrumentación manual.

- Sin embargo, se ha descrito que la instrumentación rotatoria conlleva mayores costos para implementar su uso, considerando la adquisición de un motor de endodoncia y las limas rotatorias, además de la necesidad de entrenamiento y mayor curva de aprendizaje para los tratantes ${ }^{(26)}$. Sin embargo, disminuye el tiempo de la preparación mecánica por el diseño y cinemática de las limas rotatorias, por lo que se podría optimizar el tiempo clínico del tratamiento endodóntico ${ }^{(27)}$

\section{Qué piensan los pacientes y sus tratantes}

- Dada la evidencia presentada en este resumen, la mayoría de los pacientes y tratantes podrían preferir la instrumentación rotatoria por sobre la manual debido a la menor incidencia e intensidad de dolor postoperatorio, junto con el menor uso de analgésicos postoperatorios.

- Sin embargo, algunos tratantes podrían preferir el uso de instrumentación manual, aunque implique mayor tiempo clínico, por la curva de aprendizaje que lleva la utilización de la instrumentación rotatoria sumado a los costos de éstas ${ }^{(26)}$.

- Finalmente, es importante considerar la incertidumbre de la evidencia respecto a los posibles riesgos y beneficios de la instrumentación rotatoria en comparación a la manual para que tanto pacientes como tratantes tomen una decisión informada.

\section{Diferencias entre este resumen y otras fuentes}

- Las conclusiones de este resumen sobre dolor postoperatorio coinciden con las de las revisiones identificadas, dado que concluyen que con la instrumentación rotatoria en comparación a la instrumentación manual podría existir una disminución en la incidencia de dolor y el uso de analgésicos postoperatorios.
- Las revisiones también concuerdan en la existencia de una limitación en la evidencia disponible, requiriendo un mayor número de ensayos clínicos aleatorizados, por lo que estos resultados deben ser interpretados con cautela.

- No se identificaron guías de práctica clínica que realicen recomendaciones sobre el tipo de instrumentación a utilizar en el tratamiento endodóntico. Sin embargo, la American Association of Endodontists cuenta con un suplemento que concluye que el entrenamiento previo es fundamental para la utilización de la instrumentación rotatoria en la terapia endodóntica ${ }^{(28)}$.

\section{¿Puede que cambie esta información en el futuro?}

- Futura evidencia podría cambiar las conclusiones respecto a la incidencia de dolor postoperatorio, debido a que presenta una certeza moderada. La probabilidad de que cambien las conclusiones de los desenlaces de baja y muy baja evidencia frente a nueva evidencia es alta, dada la incertidumbre existente.

- Se identificó una revisión sistemática en curso en la base de datos PROSPERO (International prospective register of systematic reviews) ${ }^{(29)}$ que podría entregar información relevante a la pregunta presentada.

- Se buscó en la International Clinical Trials Registry Platform de la Organización Mundial de la Salud y no se identificaron nuevos ensayos clínicos en curso que comparen instrumentación rotatoria y manual.

\section{CÓMO REALIZAMOS ESTE RESUMEN}

Mediante métodos automatizados y colaborativos recopilamos toda la evidencia relevante para la pregunta de interés y la presentamos en una matriz de evidencia.

Siga el enlace para acceder a la versión interactiva: Instrumentación rotatoria comparado con instrumentación manual para tratamiento endodóntico en dientes permanentes

\section{NOTAS}

Si con posterioridad a la publicación de este resumen se publican nuevas revisiones sistemáticas sobre este tema, en la parte superior de la matriz se mostrará un aviso de "nueva evidencia".

Este artículo es parte del proyecto síntesis de evidencia de Epistemonikos. Se elabora con una metodología preestablecida, siguiendo rigurosos estándares metodológicos y proceso de revisión por pares interno. Cada uno de estos artículos corresponde a un resumen, denominado FRISBEE (Friendly Summary of Body of Evidence using Epistemonikos), cuyo principal objetivo es sintetizar el conjunto de evidencia de una pregunta específica, en un formato amigable a los profesionales clínicos. Sus principales recursos se basan en la matriz de evidencia de Epistemonikos y análisis de resultados usando metodología GRADE. Mayores detalles de los métodos para elaborar este FRISBEE están descritos aquí (http://dx.doi.org/10.5867/medwave.2014.06.5997)

La Fundación Epistemonikos es una organización que busca acercar la información a quienes toman decisiones en salud, mediante el uso de tecnologías. Su principal desarrollo es la base de datos Epistemonikos (www.epistemonikos.org).

\section{DECLARACIÓN DE CONFLICTOS DE INTERESES}

Los autores declaran no tener conflictos de intereses con la materia de este artículo.

\section{AGRADECIMIENTOS}

Este resumen de evidencia fue elaborado con el apoyo metodológico del Centro Evidencia UC, Facultad de Medicina, Pontificia Universidad Católica de Chile. 


\section{Bibliografía}

1. Abbott PV. Endodontic assessment: pulps, pain and prognosis. Ann R Australas Coll Dent Surg. 2012 Apr;21:101-2. PMID: 24783841

2. Berman LH. Endodontic prognosis assessment. Alpha Omegan. 2011 Spring;104(1-2):12-7. PMID: 21905362.

3. Darcey J, Taylor C, Roudsari RV, Jawad S, Hunter M. Modern endodontic principles part 3: preparation. Dent Update. 2015 Nov;42(9):810-2, 815-8, 821-2.doi: 10.12968/ denu.2015.42.9.810. PMID: 26749789.

4. Young GR, Parashos $P$, Messer $\mathrm{HH}$. The principles of techniques for cleaning root canals. Aust Dent J. 2007 Mar;52(1 Suppl):S52-63. doi: 10.1111/j.1834-7819.2007. tb00526.x. PMID: 17546862.

5. Sun C, Sun J, Tan M, Hu B, Gao X, Song J. Pain after root canal treatment with different instruments: A systematic review and meta-analysis. Oral Dis. 2018 Sep;24(6):908-19. doi: 10.1111/odi.12854. Epub 2018 Jun 8. PMID: 29516592

6. Spohr AR, Sarkis-Onofre R, Pereira-Cenci T, Pappen FG, Morgental RD. A systematic review: Effect of hand, rotary and reciprocating instrumentation on endodontic postoperative pain. G Ital Endod. 2019; 33(2):24-34. doi: 10.32067/ gie.2019.33.02.03

7. AlRahabi MK. Predictors, prevention, and management of postoperative pain associated with nonsurgical root canal treatment: A systematic review. J Taibah Univ Med Sci. 2017 May 9;12(5):376-384. doi: 10.1016/j.jtumed.2017.03.004. PMID: 31435267

8. Del Fabbro M, Afrashtehfar KI, Corbella S, El-Kabbaney A, Perondi I, Taschieri S. In vivo and in vitro effectiveness of rotary nickel-titanium vs Manual Stainless Steel Instruments for root canal therapy: systematic review and meta-analysis. J Evid Based Dent Pract. 2018 Mar;18(1):59-69. doi: 10.1016/j.jebdp.2017.08.001. Epub 2017 Aug 19. PMID: 29478682

9. Schäfer E, Bürklein S. Impact of nickel-titanium instrumentation of the root canal on clinical outcomes: a focused review. Odontology. 2012 Jul;100(2):130-6. doi: 10.1007/ s10266-012-0066-1. Epub 2012 Apr 22. PMID: 22527909.

10. Shokraneh A, Ajami M, Farhadi N, Hosseini M, Rohani B. Postoperative endodontic pain of three different instrumentation techniques in asymptomatic necrotic mandibular molars with periapical lesion: a prospective, randomized, double-blind clinical trial. Clin Oral Investig. 2017 Jan;21(1):413-418. doi: 10.1007/s00784-016-1807-2. Epub 2016 Apr 4. PMID: 27041109

11. Talebzadeh B, Nezafati S, Rahimi S, Shahi S, Lotfi M, Ghasemi N. Comparison of manual and rotary instrumentation on postoperative pain in teeth with asymptomatic irreversible pulpitis: a randomized clinical trial. Iran Endod J. 2016 Fall;11(4):273-279. doi: 10.22037/iej.2016.4. PMID: 27790255

12. Al-Jabreen TM. Single visit endodontics: Incidence of post- operative pain after instrumentation with three different techniques: An objective evaluation study. Saudi Dent J. 2002;14:136-9.

13. Kashefinejad M, Harandi A, Eram S, Bijani A. Comparison of single visit post endodontic pain using Mtwo rotary and hand k-file instruments: a randomized clinical trial. J Dent (Tehran). 2016 Jan;13(1):10-7. PMID: 27536323

14. Ahmed M A, Dall A Q, Khoso N A, Jouhar R. Comparison of postoperative pain after Protaper rotary and manual step-back root canal preparation techniques in single visit endodontics. JPDA. 2012; 21(2):102-107.

15. Çiçek E, Koçak MM, Koçak S, Sağlam BC, Türker SA. Postoperative pain intensity after using different instrumentation techniques: a randomized clinical study. J App Oral Sci. 2017 Jan-Feb;25(1):20-26. doi: 10.1590/1678-77572016-0138. PMID:
28198972

16. Mollashahi NF, Saberi EA, Havaei SR, Sabeti M. Comparison of postoperative pain after root canal preparation with two reciprocating and rotary single-file systems: a randomized clinical trial. Iran Endod J. 2017 Winter;12(1):15-19. doi: 10.22037/ iej.2017.03. PMID: 28179917

17. Zhang WW, Jin F, Su Y. Symbol Hero642 nickel-titanium rotary instrument for single-visit root canal treatment. Journal of Clinical Rehabilitative Tissue Engineering Research. 2013 October;17(42): 7408-7413. doi: 10.3969/j.issn.20954344.2013.42.011

18. Du C, Luo X, Zhang F, Hong F. Short-term effect of two nickel titanium motors on one-time molar root canal therapy. Journal of Oral Science Research. 2016; 32: 1316-1318.

19. Dalton BC, Orstavik D, Phillips C, Pettiette M, Trope M. Bacterial reduction with nickel-titanium rotary instrumentation. J Endod. 1998 Nov;24(11):763-7. doi: 10.1016/ S0099-2399(98)80170-2. PMID: 9855830

20. Cheung GS, Liu CS. A retrospective study of endodontic treatment outcome between nickel-titanium rotary and stainless steel hand filing techniques. J Endod. 2009 Jul;35(7):938-43. doi: 10.1016/j.joen.2009.04.016. PMID: 19567311.

21. Fleming CH, Litaker MS, Alley LW, Eleazer PD. Comparison of classic endodontic techniques versus contemporary techniques on endodontic treatment success. J Endod. 2010 Mar:36(3):414-8. doi: 10.1016/j.joen.2009.11.013. Epub 2010 Jan 25. PMID: 20171354

22. Arias A, de la Macorra JC, Azabal M, Hidalgo JJ, Peters OA. Prospective case controlled clinical study of post-endodontic pain after rotary root canal preparation performed by a single operator. J Dent. 2015 Mar;43(3):389-95. doi: 10.1016/j jdent.2014.07.008. Epub 2014 Jul 17. PMID: 25038508.

23. Iqbal M, Kurtz E, Kohli M. Incidence and factors related to flare-ups in a graduate endodontic programme. Int Endod J. 2009 Feb;42(2):99-104. doi: 10.1111/j.13652591.2008.01461.x. PMID: 19134037

24. Marending M, Peters OA, Zehnder M. Factors affecting the outcome of orthograde root canal therapy in a general dentistry hospital practice. Oral Surg Oral Med Ora Pathol Oral Radiol Endod. 2005 Jan;99(1):119-24. doi: 10.1016/j.tripleo.2004.06.065 PMID: 15599359

25. Martin WJ, Ashton-James CE, Skorpil NE, Heymans MW, Forouzanfar T. What constitutes a clinically important pain reduction in patients after third molar surgery? Pain Res Manag. 2013 Nov-Dec;18(6):319-22. doi: 10.1155/2013/742468. Epub 2013 Aug 16. PMID: 23957018

26. Martins RC, Seijo MO, Ferreira EF, Paiva SM, Ribeiro Sobrinho AP. Denta students' perceptions about the endodontic treatments performed using NiTi rotary instruments and hand stainless steel files. Braz Dent J. 2012;23(6):729-36. doi: 10.1590/s0103-64402012000600018. PMID: 23338269.

27. Haapasalo M, Shen $Y$. Evolution of nickel-titanium instruments: from past to future. Endod Topics. 2013;29(1):3-17. doi: https://doi.org/10.1111/etp.12049 28. American Association of Endodontists. Rotary instrumentation: an endodontic perspective. 2008. Available from: https://endoexperience.com/documents/ Rotarylnstrumentation-AAE.pdf

29. Patnana AK, Kumar P, Singh S, Chugh A, Chugh VK. Incidence of nickle titanium file fractures in root canal treated permanent teeth - a systematic review and metaanalysis. PROSPERO 2020 CRD42020167930 Available from: https://www.crd.york. ac.uk/prospero/display_record.php?ID=CRD42020167930 